University of Wollongong

Research Online

Faculty of Social Sciences - Papers (Archive) Faculty of Arts, Social Sciences \& Humanities

2000

Visually directed walking to briefly glimpsed targets is not biased toward fixation location

John W. Philbeck

Carnegie Mellon University, johnp@uow.edu.au

Follow this and additional works at: https://ro.uow.edu.au/sspapers

Part of the Education Commons, and the Social and Behavioral Sciences Commons

Research Online is the open access institutional repository for the University of Wollongong. For further information contact the UOW Library: research-pubs@uow.edu.au 


\title{
Visually directed walking to briefly glimpsed targets is not biased toward fixation location
}

\begin{abstract}
When observers indicate the magnitude of a previously viewed spatial extent by walking without vision to each endpoint, there is little evidence of the perceptual collapse in depth associated with some other methods (eg visual matching). One explanation is that both walking and matching are perceptually mediated, but that the perceived layout is task-dependent. In this view, perceived depth beyond $2-3 \mathrm{~m}$ is typically distorted by an equidistance effect, whereby the egocentric distances of nonfixated portions of the depth interval are perceptually pulled toward the fixated point. Action-based responses, however, recruit processes that enhance perceptual accuracy as the stimulus configuration is inspected. This predicts that walked indications of egocentric distance performed without vision should exhibit equidistance effects at short exposure durations, but become more accurate at longer exposures. In this paper, two experiments demonstrate that in a well-lit environment there is substantial perceptual anisotropy at near distances $(3-5 \mathrm{~m})$, but that walked indications of egocentric distance are quite accurate after brief glimpses (150 ms), even when the walking target is not directly fixated. Longer exposures do not increase accuracy. The results are clearly inconsistent with the task-dependent information processing explanation, but do not rule out others in which perception mediates both walking and visual matches.
\end{abstract}

\section{Keywords}

fixation, location, walking, briefly, glimpsed, targets, not, visually, biased, directed, toward

\section{Disciplines}

Education | Social and Behavioral Sciences

\section{Publication Details}

Philbeck, J. W. (2000). Visually directed walking to briefly glimpsed targets is not biased toward fixation location. Perception, 29 (3), 259-272. 


\title{
Visually directed walking to briefly glimpsed targets is not biased toward fixation location
}

\author{
John W Philbeck \\ Department of Psychology, Carnegie Mellon University, Pittsburgh, PA 15213, USA; \\ e-mail: philbeck@andrew.cmu.edu \\ Received 3 August 1999, in revised form 9 December 1999
}

\begin{abstract}
When observers indicate the magnitude of a previously viewed spatial extent by walking without vision to each endpoint, there is little evidence of the perceptual collapse in depth associated with some other methods (eg visual matching). One explanation is that both walking and matching are perceptually mediated, but that the perceived layout is task-dependent. In this view, perceived depth beyond $2-3 \mathrm{~m}$ is typically distorted by an equidistance effect, whereby the egocentric distances of nonfixated portions of the depth interval are perceptually pulled toward the fixated point. Action-based responses, however, recruit processes that enhance perceptual accuracy as the stimulus configuration is inspected. This predicts that walked indications of egocentric distance performed without vision should exhibit equidistance effects at short exposure durations, but become more accurate at longer exposures. In this paper, two experiments demonstrate that in a well-lit environment there is substantial perceptual anisotropy at near distances $(3-5 \mathrm{~m})$, but that walked indications of egocentric distance are quite accurate after brief glimpses $(150 \mathrm{~ms})$, even when the walking target is not directly fixated. Longer exposures do not increase accuracy. The results are clearly inconsistent with the task-dependent information processing explanation, but do not rule out others in which perception mediates both walking and visual matches.
\end{abstract}

\section{Introduction}

When observers indicate the magnitude of spatial extents in well-structured viewing conditions, their responses often show evidence of a systematic perceptual distortion in 3-D spatial layout. A number of studies suggest that beyond relatively near distances (eg $1 \mathrm{~m}$ ), the perceived extent of intervals oriented in depth becomes increasingly foreshortened relative to that of physically equal intervals oriented in a frontoparallel plane (Baird and Biersdorf 1967; Levin and Haber 1993; Loomis et al 1992; Loomis and Philbeck 1999; Norman et al 1996; Todd et al 1995; Toye 1986; Wagner 1985). At a viewing distance of $2 \mathrm{~m}$, the relative compression is fairly small (about 15\%), while at distances of $20-40 \mathrm{~m}$ it can increase to $50 \%$ or more. These findings are remarkably robust and have been established by a variety of different behavioral tasks. One task that yields representative performance is called 'visual matching'. In a visual matching task, observers attempt to adjust the magnitude of a standard interval, oriented either in depth or in a frontoparallel plane, to match an interval oriented in some other direction (eg Baird and Biersdorf 1967; Loomis et al 1992; Norman et al 1996).

Under comparable viewing conditions, however, a different pattern emerges when observers indicate the magnitude of spatial extents by walking without vision: the dependence on viewing distance and orientation of the extent disappears (Beusmans 1998; Loomis et al 1992; Philbeck 1997). After viewing an extent, observers close their eyes and attempt to walk out to it, stopping briefly to indicate the location of each endpoint. This response has been called 'visually directed walking, a term used to emphasize the fact that although walking is directed to the location specified by vision, vision is not available while the response is being executed. By contrast, during 'visually guided walking', vision is available continuously to guide walking as it is produced. Loomis et al (1992) originally used the visually directed walking response to investigate the perception of exocentric intervals because they had been impressed with the accuracy 
with which observers indicated egocentric (or absolute) distances by walking without vision. Visually directed walking has been used extensively as an indication of egocentric distance, and accurate responding is a basic result that has been replicated many times (Corlett et al 1985; Elliott 1986; Loomis et al 1998; Philbeck and Loomis 1997; Rieser et al 1990; Sinai et al 1998; Steenhuis and Goodale 1988; Thomson 1983). Loomis et al (1992) wondered whether indications of exocentric intervals made by visually directed walking would show a similar accuracy. They found that, although there was some overwalking of the intervals, there was no anisotropy - depth and frontal intervals were overwalked by the same amount. However, when the same observers adjusted a depth extent to match a frontal extent, their visual matches showed evidence of a strong depth foreshortening.

Perceptual variables such as perceived depth, or, more generally, perceived extent, are psychological constructs that refer to internalized information about the layout of the physical world; the internal representations are commonly assumed to mediate certain behavioral measures of the analogous perceptual variables (Philbeck and Loomis 1997). The apparent inconsistency between locomotor indications of extent and visual matches of extent is remarkable for at least two reasons. First, the magnitude of the difference in responses is quite large; visual matches suggest that there is a strong perceptual foreshortening in the depth dimension (up to $50 \%$ or more), whereas compression is entirely absent in visually directed walking responses. Both types of responses have been well-studied and the results are robust across a variety of experimental settings. This being the case, large response differences between two tasks that ostensibly measure the same perceptual variable are particularly striking. Second, there has been increasing interest in the relative contributions of perceptual and nonperceptual factors in the control of spatially directed actions (Aglioti et al 1995; Brenner and Smeets 1996; Bridgeman et al 1981; Goodale et al 1986; Mack et al 1985; MacLeod and Willen 1995; Philbeck and Loomis 1997; Post and Welch 1996; Smeets and Brenner 1995). In particular, some motoric indications of spatial properties have been found to be insensitive to perceptual distortions such as those that occur in visual illusion figures. The large differences between the matching and walking responses in Loomis et al (1992) make this paradigm a useful tool for exploring processes that may underlie other apparent inconsistencies between perception and motor behavior.

\subsection{Three hypotheses}

The explanation favored by Loomis et al (1992) was that perceived egocentric distances and perceived extents are represented independently to some degree and need not be consistent. In this view, when observers perform an interval walking task, they direct their walking to two accurately perceived egocentric locations in sequence-the endpoints of the interval-without reference to the perceived exocentric separation of the endpoints that is presumably foreshortened in depth. There is experimental support for this idea, in both the visual-perception and motor-control domains (Abrams and Landgraf 1990; Baird and Biersdorf 1967; Gillam and Chambers 1985; Mack et al 1985; MacLeod and Willen 1995; Marteniuk and Roy 1972). Although this view does have explanatory power, it suggests that perceived extents are not completely specified by the perceived locations of the endpoints of those extents. This introduces considerable theoretical and computational complexity. Perhaps for this reason, investigators working within the more traditional framework have tended to conceptualize visual space as a unitary, internally consistent construct (eg Cutting and Vishton 1995; Foley 1991; Gogel 1993; Gogel and Da Silva 1987).

A second and more traditional hypothesis assumes that egocentric distances beyond 3-4 $\mathrm{m}$ are perceptually compressed, in keeping with the decreasing effectiveness of primary distance cues such as binocular parallax (Foley 1985; Gogel and Da Silva 1987). Exocentric intervals are perceptually foreshortened because they are derived 
from the perceptually compressed egocentric distances of the interval endpoints. Visual matches presumably reflect the compression in perceived egocentric distance, but the observer corrects (consciously or unconsciously) for this distortion when producing the walking responses. Thus, even though the extent judgment tasks are nominally exocentric in nature, perceived egocentric distance is the controlling input to both response types. In this view, walking and matching indications of extents appear to be inconsistent because they are calibrated differently. Although this hypothesis explains the data well for fairly simple tasks, accurate performance in more demanding tasks (eg triangulation by walking or pointing - Fukusima et al 1997; Loomis et al 1992) suggests that the details of the calibration processes are likely to be complex.

A third hypothesis is that visual matches and visually directed walking indications of extents draw upon independent spatial representations, with a relatively accurate representation underlying motoric responses and a more anisotropic representation underlying perceptual reports such as the matching judgments. Milner and Goodale (1995) have accumulated evidence from a variety of research contexts that visual information is processed independently to some degree in two anatomically distinct neural streams. They cite the Loomis et al (1992) data as behavioral evidence of this anatomical dissociation. One difficulty with this explanation as it stands is that, although walking to the first endpoint of the extent is accurate, observers typically overshoot dramatically when attempting to walk to the second endpoint; the mean systematic errors exceed $70 \%$ in some conditions (Loomis et al 1992; Philbeck 1997). Thus, although walked indications of extent are accurate in the sense of there being no evidence of depth foreshortening, they are quite inaccurate in terms of absolute error. The processes underlying this accuracy trade-off are still unclear. A complete account of the behavioral dissociation between walking and matching indications of extent may yet involve a separation of anatomical systems, but, as with the other hypotheses presented here, there are complexities that remain unexplored.

Here, a fourth alternative that sidesteps some of the problematic aspects of the other three is investigated. This explanation retains the notion of visual space as an internally consistent construct, it does not assume any corrective processes, and it assumes that a single underlying representation controls both visual matches and walking responses. The hypothesis begins with the notion that the layout of an unchanging stimulus configuration is perceived differently depending on what behavioral response is required. In this view, both matching and walking responses are based on the perceived layout, but when a matching response is required, the perceived depth is more foreshortened than it is when a walking response is required. Matching responses reflect a more immediate perception based upon the proximal stimulation, whereas walking responses reflect a more accurate perceptual representation that incorporates spatial information gathered over a relatively small epoch of time (eg several fixational eye movements). Because the perception underlying each response type depends upon the task, I will refer to this fourth alternative as the task-dependent information processing hypothesis.

Far from being a remote possibility, this hypothesis has strong theoretical underpinnings and is supported by extensive experimentation. There is abundant evidence that eye position affects perceived depth. Foley and Richards (1972) found that voluntary eye movements made between two endpoints of a depth interval can greatly increase indications of perceived depth relative to those obtained while fixation is held constant. Although the dynamics of this enhancement have not been explored, presumably the perceived depth is initially compressed and then rapidly increases to a new steady state over the course of several changes in fixation. Gogel and others (Gogel 1965; Gogel and Tietz 1977; Lodge and Wist 1968; Wist and Summons 1976) have shown that, when the eyes are held stationary and distance information is weak, the perceived depth between two target tends to be compressed: targets that are displaced in depth from a fixated 
target tend to be perceptually drawn toward the perceived distance of the fixation point. This so-called equidistance tendency (EDT) is a robust perceptual phenomenon; it is readily observed in reduced viewing conditions and is also a powerful explanatory construct in accounting for a variety of perceptual phenomena in well-structured natural environments (Gogel 1965; Gogel 1990).

Little is known about how the tasks of preparing walking versus matching responses might differentially influence the spontaneous pattern of eye movements that observers generate while they inspect a natural scene. Thus, it is possible that walking and matching tasks tend to engender different patterns of eye movements, thereby affecting perceived depth in a manner consistent with the known effects of eye position on depth perception. In this view, when observers are required to walk without vision to indicate the magnitude of an extent, they look back and forth between the endpoints of the stimulus interval. Such eye movements are to be expected; fixating the target of an intended action is a natural and spontaneous behavior and can itself enhance the stimulus information used to localize the fixated point (Ballard et al 1995; Collewijn and Erkelens 1990). At first glance, depth separations are compressed, but over the course of several eye movements the perceived extent of depth intervals becomes enhanced, in a manner consistent with the findings of Foley and Richards (1972). When observers visually compare exocentric intervals in preparation for a matching response, they presumably make fewer eye movements among the interval endpoints, causing the EDT to receive greater weight in determining the perceived endpoint separation. Thus, in the matching response, perception is in effect 'frozen' at an early time step, in which perceived depth is distorted by the EDT. In contrast, the walking response is based on a perceptual representation that has become more accurate through time by virtue of additional eye movements. Although temporal factors in the visual control of action have been discussed (Goodale et al 1994; Rossetti 1998), the dynamics of perception itself have received much less attention in the context of perceptuomotor interactions. Thus, this hypothesis remains an important theoretical possibility that is both parsimonious and accords well with existing data.

It should be mentioned that eye movements are not a crucial feature of this explanation. Gogel and Sharkey (1989) suggest that, even without eye movements, changes in voluntary attention can alter the effectiveness of the stimulus information used in determining perceived egocentric and exocentric distances. More specifically, distributing attention to particular parts of an object or configuration can result in perceptions that more accurately reflect the distal stimulation than when attention is distributed over the whole object or configuration (Rock 1986). Mack et al (1985), for example, found that observers reported larger illusions of extent in Müller-Lyer figures when their attention was distributed over the whole figure than when it was directed specifically toward the vertices. Visual matching tasks, then, could encourage the observer to distribute attention over the whole configuration, yielding distortions in perceived depth, and the walking task could focus attention specifically on the endpoints of the interval, resulting in a more accurate perception of depth.

More generally, it may be that, unless a motoric behavior such as walking is required, the benefit of additional eye movements or localized attention does not develop. If so, one would predict that depth intervals should remain perceptually foreshortened when a visual matching response is required, even if an observer spends hours inspecting a particular stimulus configuration. Consistent with this notion, nonmotoric visual comparisons of extents typically suggest a strong perceptual anisotropy, despite long periods of observation (eg Haber 1985; Levin and Haber 1993; Loomis and Philbeck 1999; Norman et al 1996; Wagner 1985).

In this paper two experiments are described. The first establishes that the foreshortening in perceived depth is robust and can be elicited even at relatively near 
viewing distances. Previous work has suggested that this was likely to be true, but this was important to confirm because near viewing distances allow a particularly powerful test of the task-dependent information processing hypothesis. After establishing that perceptual foreshortening does exist at distances of 3-5 m, that hypothesis is tested in experiment 2 by presenting stimuli at these distances. Although the phenomenon to be explained concerns differences between visually directed walking and matching indications of exocentric intervals, experiment 2 tests walking as an indication of egocentric distances. The reasons for this will be described in more detail below.

\section{Experiment 1}

Depth foreshortening has been demonstrated at relatively near distances (within $4 \mathrm{~m}$ ) under well-lit viewing conditions, but these studies typically present stimuli on a horizontal surface such as a table top, thereby creating a fairly shallow angle of regard of the viewing surface with respect to the observer's viewpoint (Baird and Biersdorf 1967; Norman et al 1996). By contrast, studies in which the observer views the stimuli on the ground from his or her natural eye height have not been conducted indoors at near distances. It is possible that perceptual anisotropy would be reduced or even eliminated under these conditions; distance information is more effective, the angle of regard is steeper, and often there are familiar-sized objects nearby that could facilitate depth scaling. It is important to confirm that perceptual anisotropy exists in this region, because relatively near viewing distances are advantageous for eliciting fixational effects. For a constant physical depth separation between two targets lying on the ground, their angular separation (seen from the viewpoint of a standing observer) becomes larger as viewing distance decreases. One might expect larger angular differences to enhance fixational effects because the angular direction of the nonfixated target is greater.

In experiment 1 , observers attempted to create depth intervals that matched frontal intervals in a natural, well-lit indoor setting. Relatively near stimulus distances $(3-5 \mathrm{~m})$ and binocular viewing were used, and angles of regard were determined by the observer's natural eye height.

\subsection{Method}

2.1.1 Participants. Eleven individuals from the university community (six male, five female) consented to participate in the study in exchange for US \$5. Their ages ranged from 18 to 27 years, with the mean age being 21 years. They all reported having normal vision in both eyes, corrected if necessary, and were naïve about the purposes of the study.

2.1.2 Design and stimuli. On each trial, the stimulus configuration consisted of three balls ( $9 \mathrm{~cm}$ in diameter), arranged in a triangular pattern on the ground. Two lay in a frontoparallel plane with respect to the observer, and were always separated by $0.5 \mathrm{~m}$. The leftmost ball of this pair lay in the observer's median sagittal plane, and the rightmost ball was displaced to the observer's right. The third ball lay behind the left ball of the frontal pair in the observer's median sagittal plane, thereby denoting a depth interval. The observer remotely adjusted the location of this ball in depth by instructing the experimenter to move it closer or farther away. The adjustment on each trial was made in either a descending or an ascending series, with each series occurring equally often. At the beginning of descending trials, the adjustable ball was placed about $1.5 \mathrm{~m}$ behind the leftmost ball of the frontal pair and moved toward the observer, and at the beginning of ascending trials, the adjustable ball was placed immediately behind the leftmost frontal ball and moved away from the observer.

The stimulus configurations were presented at each of three distances: 3,4 , and $5 \mathrm{~m}$; this was the distance from a point on the observer's instep to the near ball of the depth pair. Each combination of distance and adjustment series was measured 4 times, yielding a total of 24 trials. The presentation order was fully randomized. 
2.1.3 Laboratory. The experiment was conducted in a well-lit indoor classroom $6.0 \mathrm{~m} \times 7.7 \mathrm{~m}$. Chairs and tables were visible nearby, but there were no objects within $1.5 \mathrm{~m}$ of the stimulus configurations other than the experimenter.

2.1.4 Procedure. The observer stood throughout the experiment, and began each trial wearing a blindfold. Upon a verbal signal, he or she raised the blindfold and binocularly viewed the stimulus configuration. The experimenter grasped the adjustable ball and began to slowly move it toward or away from the observer on descending or ascending trials, respectively. To guard against the possibility that the experimenter might bias the observer's responses, the experimenter moved the ball at the same rate (about $10 \mathrm{~cm} \mathrm{~s}^{-1}$ ) on every trial until instructed to stop by the observer. Using this method of remote adjustment, the observer attempted to create a physical match between the 'depth' pair and the 'frontal' pair. The observer was allowed to continue making adjustments until fully satisfied with the match. Each trial was terminated only after the observer had viewed the stationary configuration and verified that he or she was satisfied with the match.

The instructions pointed out the distinction between the apparent separation versus the physical separation of the ball pairs, and that the apparent and physical separations can be different when configurations of objects are viewed at a slant. The observers were encouraged to take this knowledge into account and create matches such that a tape measure would yield identical values if extended between each pair of balls in sequence. Although objective instructions such as these tend to produce systematically different data from those obtained under subjective instructions, the objective-instructions data typically underestimate the magnitude of distortions in measures of perceived layout (Carlson 1977). Matches made under objective criteria thus provide a particularly strong test of the existence of perceptual distortions. Even if observers covertly adopted a more appearance-based strategy (eg creating a perceived isosceles right triangle: Foley 1972; Higashiyama 1981), one would expect these strategies to yield even greater distortions.

\subsection{Results}

Even in a well-lit indoor setting, at near distances and under binocular viewing the observers set the depth separations about 25\% larger than required to physically match the frontal separations. Figure 1 shows the average responses expressed as a ratio of the matched depth to the physical frontal separation. Two-tailed $t$-tests confirmed that, at each viewing distance, the depth settings were significantly different from the $0.5 \mathrm{~m}$ separation required to make a physical match $\left(t_{10}=4.5,5.6\right.$, and 6.6 for the 3, 4, and $5 \mathrm{~m}$ viewing distances, respectively; all $p \mathrm{~s}<0.01)$. These results strongly suggest that there was indeed sizable foreshortening in perceived depth under these conditions.

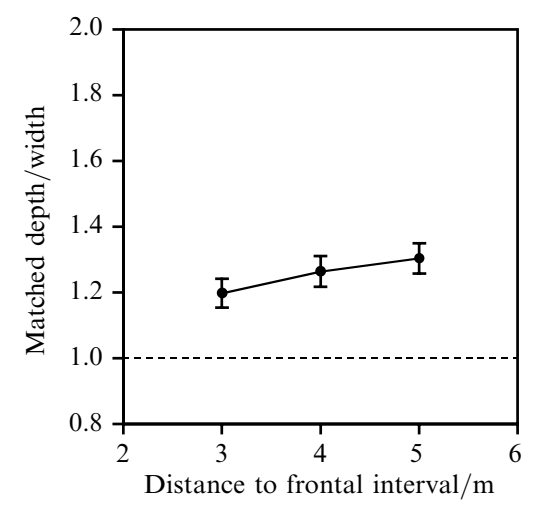

Figure 1. Results of the interval matching task of experiment 1 . The mean depth settings, divided by the standard frontal interval, are plotted as a function of the egocentric distance of the nearest element of the stimulus configuration. The data are averaged over repetition, matching series (ascending/descending), and observer. The error bars show \pm 1 standard error of the mean, and the horizontal dotted line shows the expected matching ratio under accurate responding. To facilitate comparison with previous work, the scaling of the ordinate axis is the same as that used in figures $3 \mathrm{a}$ and $5 \mathrm{a}$ of Loomis et al (1992). 


\section{Experiment 2}

After demonstrating in experiment 1 that perceived depth compression can be elicited even in relatively near space, a similar environment was used in experiment 2 to test the task-dependent information processing hypothesis. Given well-structured viewing conditions, there are two important assumptions of the task-dependent information processing hypothesis. (i) The egocentric distances of unfixated targets will be misperceived if they are based on information obtained from only one fixation, owing to the EDT. This does not require that the fixated and attended locations be coincident; Wist and Summons (1976) found that the equidistance tendency operates even when attention is directed to some location other than the fixation point. (ii) Under some circumstances, these perceptual distortions can be diminished if visual information is integrated over successive saccades or shifts in the spatial locus of attention. This predicts that if the perceived egocentric distances of unfixated points are probed before a second saccade or attention shift can extract more information, those perceived distances should be biased toward the perceived distance of the fixation point, in a manner consistent with the EDT.

Experiment 2 was conducted to test these predictions. Two balls were presented on the floor of a well-lit room, defining a depth interval. Exposure to the stimuli was limited to either $150 \mathrm{~ms}$, an interval too brief for the observer to plan and execute a saccade, or $5 \mathrm{~s}$, an interval sufficient for the execution of several saccades. In the briefexposure condition, a fixation point was presented prior to the exposure so that the observer fixated one or the other of the two balls during the exposure. After the glimpse, one of the balls was specified as the target and the task was to indicate its egocentric distance by closing the eyes and attempting to walk to its location. In the long-exposure condition, fixation was unconstrained and the task was again to walk without vision to a specified ball. If assumption (i) above is true, in the brief-exposure condition the perceived egocentric distance of the specified target ball (as measured by the walking response) should be perceptually drawn toward the perceived distance of the fixated ball; walking should be accurate when the target is directly fixated during the brief glimpse and should be biased toward the fixated ball if the walking target is not fixated during the glimpse. Assumption (ii) predicts that in the long-exposure condition walking should be accurate regardless of which ball is specified as the target, because information may now be integrated over additional saccades or shifts of attention.

Walking without vision was used as a measure of perceived distance, because in Loomis et al (1992) this response showed no compression in depth relative to frontal intervals. In previous work, my co-authors and I have found that when observers indicate the egocentric distance to a target using visually directed walking, their responses are highly consistent with verbal distance judgments, across a wide variety of viewing conditions (Loomis et al 1998; Philbeck and Loomis 1997; Philbeck et al 1997). We interpret this as strong evidence that visually directed walking is indeed responsive to perceived egocentric distance. According to the task-dependent information processing hypothesis, the cues used to control walking and visual matches should be identical at the level of a single fixation. The perceived layout that underlies the control walking, however, is assumed to change dramatically over the course of several saccades. Thus, observing the pattern of responses in the walking task is of particular interest.

Loomis et al (1992) hypothesized that visually directed walking and visual matches of exocentric intervals may produce different results because walking is directed to two endpoint locations in succession. This suggests that egocentric information receives greater weight than exocentric information in determining the final stopping points in the walking task, even though the task is nominally exocentric. In an attempt to shed some light on this matter, observers in the current experiment viewed the stimuli either with both eyes or with one eye covered. Because closing one eye eliminates binocular 
disparity, a powerful cue to perceived depth, the separation between the targets should appear smaller and might therefore enhance equidistance effects.

\subsection{Method}

3.1.1 Observers. Eight observers (four men and four women) from the university community were paid $\$ 16$ to participate in this study. Their ages ranged from 20 to 28 years; the median age was 23.5 years. Five of the eight observers were within normal limits on tests of stereoacuity and near and far visual acuity, corrected if necessary; two others had poorer stereoacuity (thresholds of 29.44 and $33.06 \mathrm{~s}$ of arc) but were otherwise within normal limits. All observers were naïve about the purpose of the experiment and the stimulus locations to be used.

3.1.2 Design and stimuli. The observers participated in the monocular and binocular blocks on different days, separated by about 1 week. The block order was counterbalanced and equal numbers of men and women participated in each order. The exposure duration manipulation $(150 \mathrm{~ms}, 5 \mathrm{~s})$ was blocked, and the block order was counterbalanced. The exposure duration block order for each observer was the same for both monocular and binocular sessions. Effects of gender and block order were not analyzed.

The experiment was conducted in a well-lit indoor classroom, $7.3 \mathrm{~m} \times 7.9 \mathrm{~m}$ (see figure 2). The stimuli were two balls $(12.4 \mathrm{~cm}$ diameter), presented on the floor in the

(a)

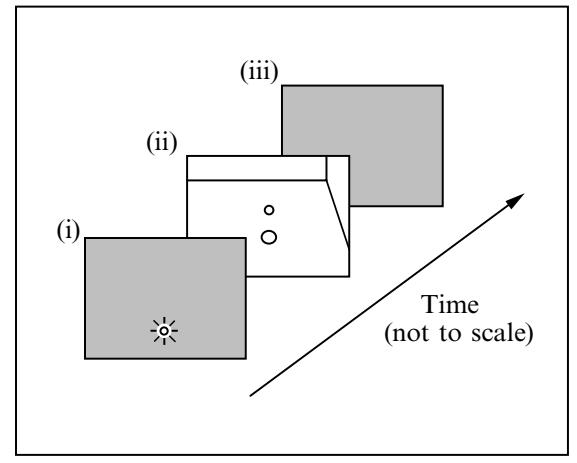

Possible

target

locations

Virtual location of illuminated lamp

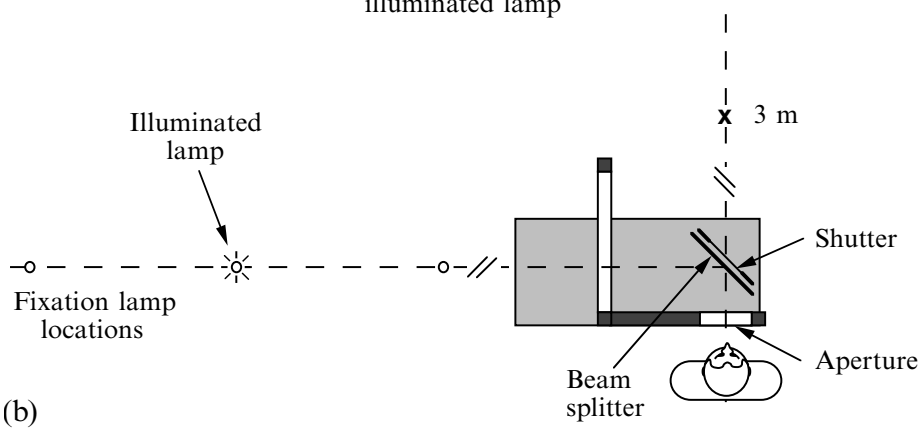

(b)

Figure 2. (a) Schematic diagram of the temporal sequence of views seen by the observer in a typical brief-exposure trial of experiment 2. (i) As the trial begins, a fixation point appears for $4 \mathrm{~s}$ while the shutter is opaque. (ii) The fixation point is extinguished, and immediately thereafter the shutter becomes transparent for $150 \mathrm{~ms}$. (iii) The shutter becomes opaque once more, after which the walking target is specified and the walking response is executed. (b) Schematic drawing of a portion of the laboratory space, showing the locations of the fixation lamps, the three possible target locations, and the beam splitter/shutter apparatus. One fixation lamp is shown to be illuminated, to illustrate the correspondence between the location of the virtual image of the lamp and the associated target location. 
observer's median sagittal plane. On each trial, these balls could appear at one of three equally likely pairings of locations: 3 and $4 \mathrm{~m}, 4$ and $5 \mathrm{~m}$, and 3 and $5 \mathrm{~m}$. These separations were chosen because they were in the range of those used by Loomis et al (1992). The near and far ball of these pairs were each selected as the walking target on half the trials. In the $150 \mathrm{~ms}$ exposure trials, observers fixated one of the two balls during the exposure, with equal frequency. The combination of fixation location and walking target meant that observers ultimately attempted to walk to either a fixated or an unfixated stimulus. Note that when a particular walking target was directly fixated, it was always seen in the context of a non-target stimulus: eg a fixated walking target at $3 \mathrm{~m}$ was seen in the context of another ball at either $4 \mathrm{~m}$ or $5 \mathrm{~m}$. Variations in the location of the unfixated, non-target ball associated with a particular walking target was not a manipulation of interest and it in fact had little effect on the data, so the data were collapsed across this variable. In the $5 \mathrm{~s}$ exposure conditions, fixation was unconstrained, so fixation location could not be completely crossed across exposure duration. Two measurements were obtained for each condition, and within each block the presentation order was fully randomized.

3.1.3 Apparatus. To view the stimuli, the observer stood behind a wooden frame that housed a liquid-crystal shutter and beam splitter. From the observation position, the frame had the appearance of a door with a horizontal rectangular aperture $(12.7 \mathrm{~cm} \times 17.8 \mathrm{~cm})$ in it. The height between the ground and the center of the aperture was $147 \mathrm{~cm}$ for all observers; this ensured that the angular separation between targets in each condition was the same for all observers.

Just behind the aperture was a liquid-crystal shutter (Edmund Scientific: New Jersey) used to occlude vision of the classroom and targets. The shutter could be made transparent or opaque with nominal rise and fall times of 10 and $30 \mathrm{~ms}$, respectively. When the shutter was transparent, the observer could see the stimulus balls on the floor of the classroom. When the shutter was opaque, it blocked the view of the classroom and had the appearance of a featureless gray field. A piece of clear glass behind the shutter functioned as a beam splitter to superimpose the image of the fixation point onto the appropriate stimulus location. Each fixation point was generated by one of three small incandescent lamps $(12 \mathrm{~V}, 80 \mathrm{~mA})$ secured to the floor to the observer's left. At the beginning of the brief-exposure trials, the reflected image of one of the fixation lamps appeared as a luminous pinpoint within the gray field of the opaque shutter, in a location that was optically coincident (in egocentric distance as well as in direction) with one of the three possible target locations. Because the shutter material was itself slightly reflective and not entirely flat, a dim 'halo' image of the fixation lamp appeared to surround the sharp image reflected off the beam splitter; the observers were instructed to ignore the halo. The fixation lamps were not visible when extinguished. It may be that the perceived distance of a particular fixation lamp and its corresponding stimulus-ball location differed somewhat, owing to the fact that the stimulus balls were seen in the context of the well-lit classroom and the fixation lamps were seen in the context of the gray field of the opaque shutter. However, one would expect the more reduced viewing context surrounding the fixation point to enhance the influence of the EDT.

3.1.4 Procedure. One practice trial (without error feedback) was conducted to familiarize the observer with the procedure. Observers wore hearing protectors throughout the experiment to minimize auditory localization cues. An eye patch was worn over the nondominant eye during the monocular session.

$150 \mathrm{~ms}$ exposure trials. At the beginning of each trial, one of the fixation lamps was illuminated against the opaque shutter for $4 \mathrm{~s}$ and then extinguished, followed immediately by a $150 \mathrm{~ms}$ glimpse of the classroom. After this, the observer lowered the blindfold and the experimenter verbally specified which ball was to be the walking 
target ('near ball' or 'far ball'). The observer sidestepped one pace to the right to maneuver around the frame, and attempted to walk to the physical location of the specified target (under objective instructions). The walked distance was measured as the straight-line distance from a point directly beneath the viewing aperture to the observer's left instep at the terminal location of the walking response. The observer was led back to the starting point without vision and no error feedback was given.

5 s exposure trials. These trials were conducted in much the same way as the $150 \mathrm{~ms}$ trials, except that no fixation lamp was illuminated. At the beginning of each trial, the shutter was made transparent, providing a $5 \mathrm{~s}$ glimpse of the room and target stimuli. As in the brief-exposure trials, the experimenter specified the walking target after the observer's vision had been obscured, and then the observer executed the response.

\subsection{Results}

Walked distances were essentially accurate in all conditions, showing an average constant error of about $6.5 \%$ (undershooting) of the target distance. The manipulation of exposure duration had very little effect upon walked distance, and within the brief-exposure conditions there was no effect of fixation location. Similarly, there was no effect of number of eyes used during viewing.

The left-hand panels of figure 3 show the pattern of responses that would be predicted if the EDT biased walking toward the fixated point. In the leftmost top panel, walking
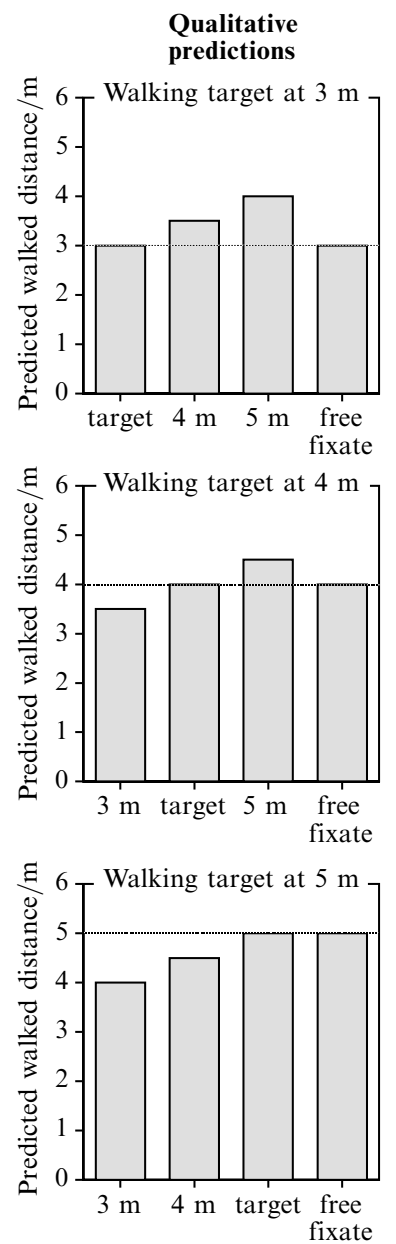

Fixation location
Results
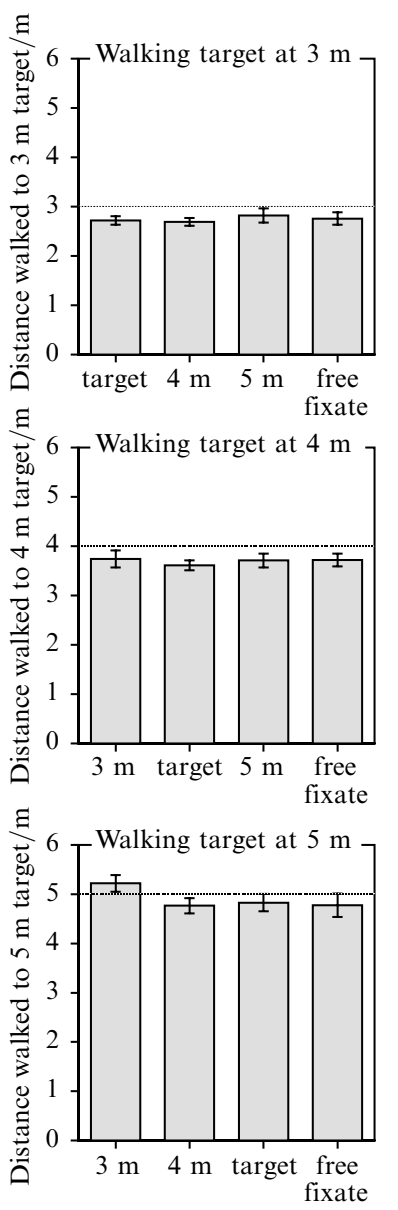

Fixation location
Figure 3. Left-hand panels. Qualitative predictions for the effect of the equidistance tendency on walked indications of egocentric target distance executed without vision. When fixation is controlled by brief stimulus presentation, the perceived distance of the unfixated point, as measured by visually directed walking, is predicted to be biased toward the perceived distance of the fixation point, which is assumed to be accurate. The perceived target distance is also predicted to be accurate under longer exposure durations (bars marked 'free fixate'), in which fixation is unrestricted. The magnitudes of biases in this figure were chosen to illustrate the predicted ordering of responses if an equidistancetendency-like bias were operating and do not represent more quantitative predictions.

Right-hand panels. Mean walked distances in the brief exposure trials (the three leftmost bars in each panel) and the long exposure trials (bars marked 'free fixate' in each panel). The figures collapse across the monocular/binocular viewing manipulation. The data for the bars marked 'target' and 'free fixate' were collapsed over variations in non-target stimulus locations. The error bars show \pm 1 standard error of the mean. 
is predicted to be directed accurately to the walking target when it is directly fixated (leftmost bar). The second bar from the left shows a condition in which the targets are at 3 and $4 \mathrm{~m}$. The target at $4 \mathrm{~m}$ is fixated during the glimpse, and then the $3 \mathrm{~m}$ target is designated as the walking target. The EDT predicts that the $3 \mathrm{~m}$ target should be overshot because the perceived location of the $3 \mathrm{~m}$ target is drawn toward that of the fixated target at $4 \mathrm{~m}$. In the third bar, the walking target is again $3 \mathrm{~m}$ but the fixated location is at $5 \mathrm{~m}$. The perceived distance of the $3 \mathrm{~m}$ target is predicted to be drawn toward the fixated target at $5 \mathrm{~m}$, yielding even more overshooting. Walking is predicted to be accurate in the long-exposure condition (fourth bar), because there is sufficient time to make additional saccades between the interval endpoints. The predictions for walking directed to the other two stimulus locations follow similar logic. For purposes of illustration, the perceived distance of the unfixated point (as measured by visually directed walking) is shown to be perturbed by $50 \mathrm{~cm}$ for every $1 \mathrm{~m}$ increase in the physical separation of the fixated and unfixated stimulus balls. This degree of perturbation was chosen merely to illustrate the predicted ordering of responses and is not meant to represent quantitative predictions of the effect of fixation location on perceived distance.

The right-hand panels of figure 3 show the results of the $150 \mathrm{~ms}$ exposure trials (the three leftmost bars in each panel) and the $5 \mathrm{~s}$ exposure trials (the rightmost bar in each panel). This figure collapses across the monocular/binocular manipulation because, as we will see, statistical analyses showed this manipulation to have no effect. The bars marked 'target' represent responses in trials in which the walking target was directly fixated during the brief glimpse, and those marked 'free fixate' represent responses in which fixation was unconstrained by virtue of the longer exposure duration. The data in these bars were collapsed across variations in the non-target stimulus location, as mentioned in section 3.1.2. The other bars in figure 3 show trials in which one ball was fixated but the other ball was subsequently specified as the walking target. A comparison of the predicted pattern of responses in figure 3 (left-hand panels) with the actual data (right-hand panels) shows that manipulation of the fixation location did not yield the pattern of results predicted by the task-dependent information processing hypothesis. None of the manipulations, including fixation location, affected the precision of responses; the variable error, both between and within observers, was about $6 \%$ of the target distance for all conditions.

Although these results are readily apparent in the figure, some sort of statistical comparison is needed. First, note that the fixation manipulation (foveal/parafoveal) could not be completely crossed with exposure duration $(150 \mathrm{~ms} / 5 \mathrm{~s})$, because eye movements were unconstrained in the $5 \mathrm{~s}$ exposure trials. To deal with this, the walking responses to each target distance were analyzed in separate ANOVAs, with the unconstrained fixation in the $5 \mathrm{~s}$ exposure block treated as one of three levels of the fixation manipulation (foveal/parafoveal/unconstrained). The data were averaged over the two measurements per condition prior to performing the ANOVAs. (A separate ANOVA was performed on the $150 \mathrm{~ms}$ trials by themselves, with target distance included as a variable, but the results of this analysis were not substantially different from the one mentioned above, so those results will not be included here.)

A primary assumption of the task-dependent information processing hypothesis is that the locations of unfixated targets will be distorted by the EDT if they are based on information obtained from only one fixation; specifically, the perceived egocentric distance of an unfixated target should be displaced toward the perceived egocentric distance of the fixated point (Gogel 1965). The data did not support this prediction: there was no main effect for fixation $\left(F_{2,14}=0.098,1.116\right.$, and 2.377 for 3,4 , and $5 \mathrm{~m}$ viewing distances; all $p \mathrm{~s}>0.05)$. Similarly, there was no main effect of presence or absence of binocular disparity $\left(F_{1,7}=0.207,0.501\right.$, and 0.108 for 3 , 4, and $5 \mathrm{~m}$ viewing conditions; 
$p>0.05)$, and no disparity $\times$ fixation interaction $\left(F_{2,14}=1.233,0.252\right.$, and 0.431 for 3 , 4 , and $5 \mathrm{~m}$ viewing conditions; $p>0.05$ ).

\subsection{Discussion}

Task-dependent information processing does not provide a good explanation of the differences between the visual matching and walking indications of spatial intervals in Loomis et al (1992). As measured by visually directed walking, the perceived location of unfixated targets was not drawn toward the perceived distance of the fixated location when vision was limited to a single fixation. Thus, there is no evidence that the EDT biased walking under the well-lit viewing conditions of this experiment. It is interesting that a $150 \mathrm{~ms}$ exposure to the stimulus scene was sufficient to yield nearly accurate walking performance. Because of the high walking accuracy under brief stimulus exposures, it remains unclear whether additional saccades might enhance the accuracy of perceived locations, if distortions are present. Walking without vision did not indicate a further change in perceived location under longer viewing exposures.

Even though experiment 2 suggests that inappropriate fixation does not alter the perceived distance of the endpoints of a depth interval, this does not mean that the depth interval itself was accurately perceived. Although explicit judgments of the magnitude of exocentric intervals were not obtained here, other research suggests that, under similar viewing conditions, the perceived extent of an interval is not solely a function of the perceived egocentric distances of the endpoints defining the interval. Loomis and Philbeck (1994) found that an alteration of stimulus cues (monocular vs binocular viewing) that produced a change in perceived depth as measured by a matching task had little or no effect upon perceived egocentric distance indicated by open-loop point-ing and walking. To the extent that the matching and open-loop motoric behaviors were in fact responsive to perceived extents and distances, these results provide evidence in favor of some degree of dissociation of perceived extents and perceived locations. It remains to be seen, however, whether this apparent dissociation also interacts with response modality (vision-based or action-based).

What, then, is the explanation for the differences between the visual matches and walked indications of extent in Loomis et al (1992)? The experiments presented here provide strong evidence that the differences are not due to an equidistance effect, but they do not rule out any of the three other hypotheses discussed in the introduction. As was mentioned earlier, however, each of these other hypotheses requires further elaboration to account for all the data. Clearly, this is a complex issue, and a satisfying explanation may ultimately draw from more than one of these hypotheses. Given the number of alternatives, however, the work presented here makes a substantial contribution by ruling out a particularly parsimonious explanation that is supported by much theoretical and empirical evidence.

Acknowledgements. This research was supported by ONR Grant N00014-95-1-0573 to Jack M Loomis. Portions of the data were reported at the 1997 annual meeting of the Association for Research in Vision and Ophthalmology. The author thanks Jack M Loomis, Marlene Behrmann, John M Foley, David H Brainard, and Mary Hegarty for many useful comments on earlier versions of the manuscript, Walter C Gogel for helpful discussions, and Shira Brill for her assistance in conducting the experiment.

\section{References}

Abrams R A, Landgraf J Z, 1990 "Differential use of distance and location information for spatial localization" Perception \& Psychophysics 47349 - 359

Aglioti S, DeSouza J F, Goodale M A, 1995 "Size-contrast illusions deceive the eye but not the hand" Current Biology $5679-685$

Baird J C, Biersdorf W R, 1967 "Quantitative functions for size and distance judgments" Perception \& Psychophysics $2161-166$ 
Ballard D H, Hayhoe M M, Pelz J B, 1995 "Memory representations in natural tasks" Journal of Cognitive Neuroscience 7 66-80

Beusmans J M H, 1998 "Optic flow and the metric of the visual ground plane" Vision Research $381153-1170$

Brenner E, Smeets J B, 1996 "Size illusion influences how we lift but not how we grasp an object" Experimental Brain Research $111473-476$

Bridgeman B, Kirch M, Sperling A, 1981 "Segregation of cognitive and motor aspects of visual function using induced motion" Perception \& Psychophysics 29 336-342

Carlson V R, 1977 "Instructions and perceptual constancy judgments", in Stability and Constancy in Visual Perception: Mechanisms and Processes Ed.W Epstein (New York: John Wiley) pp $217-254$

Collewijn H, Erkelens C J, 1990 "Binocular eye movements and the perception of depth", in Eye Movements and Their Role in Visual and Cognitive Processes Ed. E Kowler (Amsterdam: Elsevier) pp $213-261$

Corlett J T, Patla A E, Williams J G, 1985 "Locomotor estimation of distance after visual scanning by children and adults" Perception $14257-263$

Cutting J E, Vishton P M, 1995 "Perceiving layout and knowing distances: The integration, relative potency, and contextual use of different information about depth", in Handbook of Perception and Cognition volume 5 Perception of Space and Motion Eds W Epstein, S J Rogers (San Diego, CA: Academic Press) pp 69-117

Elliott D, 1986 "Continuous visual information may be important after all: A failure to replicate Thomson (1983)" Journal of Experimental Psychology: Human Perception and Performance $12388-391$

Foley J M, 1972 "The size-distance relation and intrinsic geometry of visual space: Implications for processing" Vision Research $12323-332$

Foley J M, 1985 "Binocular distance perception: egocentric distance tasks" Journal of Experimental Psychology: Human Perception and Performance 11 133-149

Foley J M, 1991 "Binocular space perception", in Binocular Vision Ed. D Regan (Boca Raton, FL: CRC Press) pp $75-92$

Foley J M, Richards W, 1972 "Effects of voluntary eye movement and convergence on the binocular appreciation of depth" Perception \& Psychophysics $11423-427$

Fukusima S S, Loomis J M, Da Silva J A, 1997 "Visual perception of egocentric distance as assessed by triangulation" Journal of Experimental Psychology: Human Perception and Performance $2386-100$

Gillam B, Chambers D, 1985 "Size and position are incongruous: Measurements on the MüllerLyer figure" Perception \& Psychophysics $37549-556$

Gogel W C, 1965 "Equidistance tendency and its consequences" Psychological Bulletin $64153-163$

Gogel W C, 1990 "A theory of phenomenal geometry and its applications" Perception \& Psychophysics $\mathbf{4 8} 105-123$

Gogel W C, 1993 "The analysis of perceived space", in Foundations of Perceptual Theory. Advances in Psychology Ed. S C Masin (Amsterdam, Netherlands: Elsevier) pp 113-182

Gogel W C, Da Silva J A, 1987 "A two-process theory of the response to size and distance" Perception \& Psychophysics $41220-238$

Gogel W C, Sharkey T J, 1989 "Measuring attention using induced motion" Perception 18 303-320

Gogel W C, Tietz J D, 1977 "Eye fixation and attention as modifiers of perceived distance" Perceptual \& Motor Skills $45343-362$

Goodale M A, Jakobson L S, Keillor J M, 1994 "Differences in the visual control of pantomimed and natural grasping movements" Neuropsychologia $321159-1178$

Goodale M A, Pelisson D, Prablanc C, 1986 "Large adjustments in visually guided reaching do not depend on vision of the hand or perception of target displacement" Nature (London) $320748-750$

Haber R N, 1985 "Toward a theory of the perceived spatial layout of scenes" Computer Vision, Graphics, and Image Processing $31282-321$

Higashiyama A, 1981 "Variation of curvature in binocular visual space estimated by the triangle method" Vision Research 21925 -933

Levin C A, Haber R N, 1993 "Visual angle as a determinant of perceived interobject distance" Perception \& Psychophysics $\mathbf{5 4} 250-259$

Lodge H, Wist E R, 1968 "The growth of the equidistance tendency over time" Perception \& Psychophysics 3 97-103 
Loomis J M, Da Silva J A, Fujita N, Fukusima S S, 1992 "Visual space perception and visually directed action" Journal of Experimental Psychology: Human Perception and Performance 18 $906-921$

Loomis J M, Klatzky R L, Philbeck J W, Golledge R, 1998 "Assessing auditory distance perception using perceptually directed action" Perception \& Psychophysics 60966 -980

Loomis J M, Philbeck J W, 1994 "Distortion of visual space under full cues is not scale invariant", paper presented at the Annual Meeting of the Psychonomic Society, St Louis, MO, November

Loomis J M, Philbeck J W, 1999 "Is the anisotropy of perceived 3-D shape invariant across scale?" Perception \& Psychophysics $61397-402$

Mack A, Heuer F, Villardi K, Chambers D, 1985 "The dissociation of position and extent in Müller-Lyer figures" Perception \& Psychophysics 37335 - 344

MacLeod D I A, Willen J D, 1995 "Is there a visual space?", in Geometric Representations of Perceptual Phenomena: Papers in Honor of Tarow Indow on his 70th Birthday Eds R D Luce, M D’Zmura, D D Hoffman, G J Iverson, A K Romney (Mahwah, NJ: Lawrence Erlbaum Associates) pp $47-60$

Marteniuk R G, Roy E A, 1972 "The codability of kinesthetic location and distance information" Acta Psychologica $36471-479$

Milner A D, Goodale M A, 1995 The Visual Brain in Action (Oxford: Oxford University Press)

Norman J F, Todd J T, Perotti V J, Tittle J S, 1996 "The visual perception of three-dimensional length" Journal of Experimental Psychology: Human Perception and Performance 22 173-186

Philbeck J W, 1997 Processes Underlying Apparently Paradoxical Indications of Extent unpublished doctoral dissertation, University of California, Santa Barbara, CA

Philbeck J W, Loomis J M, 1997 "Comparison of two indicators of perceived egocentric distance under full-cue and reduced-cue conditions" Journal of Experimental Psychology: Human Perception and Performance $2372-85$

Philbeck J W, Loomis J M, Beall A C, 1997 "Visually perceived location is an invariant in the control of action" Perception \& Psychophysics $59601-612$

Post R B, Welch R B, 1996 "Is there dissociation of perceptual and motor responses to figural illusions?" Perception $25569-581$

Rieser J J, Ashmead D H, Talor C R, Youngquist G A, 1990 "Visual perception and the guidance of locomotion without vision to previously seen targets" Perception 19 675-689

Rock I, 1986 "The description and analysis of object and event perception", in Handbook of Perception and Human Performance volume 2, Eds K R Boff, L Kaufman, J P Thomas (New York: John Wiley) pp $331-371$

Rossetti Y, 1998 "Implicit short-lived motor representations of space in brain damaged and healthy subjects" Consciousness and Cognition $7520-558$

Sinai M J, Ooi T L, He Z J, 1998 "Terrain influences the accurate judgement of distance" Nature (London) $395497-500$

Smeets J B J, Brenner E, 1995 "Perception and action are based on the same visual information: distinction between position and velocity" Journal of Experimental Psychology: Human Perception and Performance 21 19-31

Steenhuis R E, Goodale M A, 1988 "The effects of time and distance on accuracy of targetdirected locomotion: Does an accurate short-term memory for spatial location exist?" Journal of Motor Behavior $20399-415$

Thomson J A, 1983 "Is continuous visual monitoring necessary in visually guided locomotion?" Journal of Experimental Psychology: Human Perception and Performance 9 427-443

Todd J T, Tittle J S, Norman J F, 1995 "Distortions of three-dimensional space in the perceptual analysis of motion and stereo" Perception 24 75-86

Toye R C, 1986 "The effect of viewing position on the perceived layout of space" Perception \& Psychophysics $\mathbf{4 0} 85-92$

Wagner M, 1985 "The metric of visual space" Perception \& Psychophysics 38 483-495

Wist E R, Summons E, 1976 "Spatial and fixation conditions affecting the temporal course of changes in perceived relative distance" Psychological Research 39 99-112 